OPEN ACCESS

Edited by:

Antonio Caggiano,

Darmstadt University of Technology,

Germany

Reviewed by:

Juan Xie,

Changsha University of Science and

Technology, China

Józef Haponiuk,

Gdansk University of Technology,

Poland

Xiaoming Huang,

Southeast University, China

*Correspondence:

Hengxiao Xue

xhx1570@126.com

Specialty section:

This article was submitted to

Structural Materials,

a section of the journal

Frontiers in Materials.

Received: 29 October 2020

Accepted: 22 December 2020

Published: 02 February 2021

Citation:

Xue H, Cao Y, Liu Q, Zhang H and Zhang M (2021) Stability Evaluation and Mechanism of Asphalts Modified

With Various Rubber

Powder Contents.

Front. Mater. 7:622479.

doi: $10.3389 /$ fmats.2020.622479

\section{Stability Evaluation and Mechanism of Asphalts Modified With Various Rubber Powder Contents}

\author{
Hengxiao Xue ${ }^{1 *}$, Yuanbo Cao ${ }^{1}$, Qi Liu ${ }^{2}$, Hongfei Zhang ${ }^{1}$ and Mingliang Zhang ${ }^{1}$ \\ ${ }^{1}$ College of Highways, Chang'an University, Xi'an, China, ${ }^{2}$ School of Transportation, Southeast University, Nanjing, China
}

Ground tires are one of the main sources of urban solid waste. Rubber powder-modified asphalt provides an effective method to solve the problem, and it presents good high- and low-temperature performance in sustainable pavement construction. However, the storage properties of rubber powder-modified asphalts prepared from the traditional low-temperature shear mixing method are unstable, which restricts their application. In this study, four test methods (the softening-point test; the dynamic shear rheological test; the Laboratory Asphalt Stability Test; and fluorescence image analysis) and six evaluation

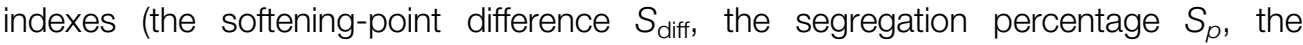
segregation index $I_{\text {se }}$, the segregation rate $R_{s}$, the degradation rate $R_{d}$, and the stability index $I_{\mathrm{st}}$ ) were employed to analyze the storage abilities of asphalts modified by various activated rubber powder contents. The results show that the storage properties have a positive correlation with rubber powder content in the modified asphalt, and a rubber powder content of $60 \%$ is proven to be the optimum mixing amount. The rubber powders can distribute uniformly in the asphalt matrix when mixed below the optimum mixing amount; otherwise a rubber powder agglomeration is formed. The quantitative morphology analysis results are in good agreement with the laboratory test conclusions. The activated rubber powder shows better compatibility compared with ordinary rubber powders and presents a promising method to treat waste ground tires.

Keywords: rubber powder-modified asphalts, stability evaluation, mechanism, sustainable pavement, fluorescence imaging method

\section{INTRODUCTION}

Waste tires are significant contributors to urban solid waste (Siddika et al., 2019; Luo et al., 2020). In pavement construction, rubber powder-modified asphalt presents good performance in both high- and low-temperature working conditions (Ding et al., 2019), and it provides a sustainable solution to the solid waste problem (Ma et al., 2017; Zhang et al., 2020). However, due to the compatibility problem between rubber powder and asphalt (Li et al., 2019), the storage ability of modified asphalt has drawn much attention in sustainable pavement construction (Guo et al., 2020; Ma et al., 2020). The main factors affecting the storage properties of modified asphalts include the properties of asphalts and rubber powders and the compatibility between them $\mathrm{Yu}$ et al., 2018). Therefore, an effective method to improve the storage stability of rubber powdermodified asphalt is to activate the surface of rubber powder (Liao and Li, 2004; Yu et al., 2018; Li et al., 2019). The light component in asphalt may also affect the distribution properties of rubber powder (Sienkiewicz et al., 2017). 
Recently, much attention has been paid to the storage stability of rubber powder-modified asphalt (Bahia and Zhai, 2000). The segregation test provides an evaluation method to assess the storage ability of modified asphalt. The test is easily performed and has low cost (Zhong-Yang et al., 2018). The National Highway Cooperation Research Program (NCHRP) of the United States has developed a new Laboratory Asphalt Stability Test (LAST) to evaluate the stability of modified asphalt based on the segregation test (Bahia and Zhai, 2000). Li Ping developed a Modified Asphalt Stability Test (MAST) on the basis of the LAST (Li, 2005). Yin Jiming evaluated the storage stability of modified asphalt with LH-1 stabilizer utilizing the MAST method and test-tube method (Yin, 2008).

Chemical and physical modifications are the two main methods to improve the stability of rubber powder-modified asphalt (Artamendi et al., 2006; Zhang et al., 2017). The physical method is to diminish the rubber powder particles at high temperature and high-speed shearing condition (Maccarrone, 1991); the compatibility between rubber powder and asphalt can be improved by increasing the specific surface area (Shi, 2011). The chemical method is by activating the surface of rubber powder or adding stabilizer. The activated rubber powder has better surface activity in the asphalt matrix, which makes it easier to disperse during shear mixing (Mu et al., 2020).

Research shows that rubber powder-modified asphalt prepared by the activation of modified rubber powder has lower viscosity and better stability in the same condition compared with ordinary rubber powder ( $\mathrm{Lu}$ et al., 2014; Ma et al., 2017; Hallmark-Haack et al., 2019). Recently, the terminal blend (TB) method has been widely accepted as a means of modifying asphalts with rubber powder. The high-speed loading treatment was applied on base asphalt after mixing with highcontent rubber powder at high temperature (Tan et al., 2020). The TB method ensures that the rubber powders have good distribution properties after fully swelling. The depolymerization level of rubber powder is increased significantly; hence, the storage stability of modified asphalt can be improved (Presti, 2013).

To summarize the previous research achievements, the segregation test method, the LAST method, and the dynamic mechanical method were employed to assess the storage stability of modified asphalts in this study. To further research the storage stability mechanism, fluorescence microscope (FM) technology was used to scan the micro-properties of modified asphalt. The correlation between the laboratory test results and mechanism of storage properties of rubber powdermodified asphalt can be established, which could provide a reference for improving the service ability of sustainable pavements.

\section{TESTS AND METHODS}

\section{Materials}

The 40-mesh activated rubber powder was pre-swelled with $20 \%$ waste oil. The microwave treatment on rubber powder was selected as $2 \mathrm{~min}$ because toxic gases were produced after $2 \mathrm{~min}$, and the rubber powders started to burn (Yin et al., 2021). The rubber powders were obtained from the waste meridian tire plant of Tianjin City, and the detailed properties of the rubber powder can be seen in Table 1. Filtered motor oil was employed as the waste oil in this study, with a density of $0.89 \mathrm{~g} / \mathrm{cm}^{3}$. To prepare the modified asphalt, the shearing temperature is selected as $230-240^{\circ} \mathrm{C}$, the shearing rate is $3,000 \mathrm{r} / \mathrm{min}$, and the shearing time is $5 \mathrm{~h}$. The asphalts used were SK-90\# from South Korea, and the properties of the asphalt can be seen in Table 2 .

\section{Evaluation Methods Segregation Test}

The segregation test (T0661-2011) is the most widely used method to evaluate the storage stability of polymer-modified asphalt. According to the specification of "Standard Test Methods of Bitumen and Bituminous Mixtures for Highway Engineering" (JTG E20-2011, 2011), the testing procedure can be summarized briefly as below:

(1) The asphalt sample is heated at the recommended temperature, and then $50 \pm 0.5 \mathrm{~g}$ of asphalt is poured into the separation tube.

(2) The segregation tube is conditioned vertically in the oven for $48 \pm 1 \mathrm{~h}$ at $163 \pm 5^{\circ} \mathrm{C}$.

(3) After heating and segregation, the separation tube is removed together with the test tube rack and placed into a freezer vertically for cooling for at least $4 \mathrm{~h}$ to make the asphalt sample solidify.

(4) The separation tube is cooled in a temperature-controlled box for $4 \mathrm{~h}$ until the solidification of asphalt.

TABLE 1 | Properties of waste rubber powder tires.

\begin{tabular}{|c|c|c|c|c|}
\hline & Properties & American standard & Chinese standard & Actual value \\
\hline \multirow[t]{4}{*}{ Physical index } & Relative density $\left(\mathrm{g} / \mathrm{cm}^{3}\right)$ & $1.1-1.2$ & $1.1-1.3$ & 1.26 \\
\hline & Moisture content (\%) & $\leq 0.75$ & $<1$ & 0.83 \\
\hline & Metal content (\%) & $<0.1$ & $<0.03$ & 0.02 \\
\hline & Fiber content (\%) & -- & $<1$ & 0.8 \\
\hline \multirow[t]{5}{*}{ Chemical index } & Acetone extract (\%) & $\leq 25$ & $\leq 22$ & 10.55 \\
\hline & Ash content (\%) & $\leq 8$ & $\leq 8$ & 2.43 \\
\hline & Carbon black content (\%) & $20-40$ & $\geq 28$ & 34.78 \\
\hline & Rubber hydrocarbon content (\%) & $40-55$ & $\geq 42$ & 52.24 \\
\hline & Natural rubber content (\%) & $16-24$ & $\geq 30$ & 30.56 \\
\hline
\end{tabular}


TABLE 2 | Properties of SK-90\# asphalt.

\begin{tabular}{|c|c|c|c|}
\hline \multicolumn{2}{|l|}{ Properties } & Specification value & SK-90\# \\
\hline \multicolumn{2}{|c|}{ The penetration $\left(25^{\circ} \mathrm{C}, 100 \mathrm{~g}, 5 \mathrm{~s}\right) / 0.1 \mathrm{~mm}$} & $80-100$ & 89.9 \\
\hline \multicolumn{2}{|c|}{ Softening point $/{ }^{\circ} \mathrm{C}$} & $\nless 42$ & 45.5 \\
\hline \multicolumn{2}{|c|}{ Ductility $\left(5 \mathrm{~cm} / \mathrm{min}, 10^{\circ} \mathrm{C}\right) / \mathrm{cm}$} & $\nless 100$ & $>100$ \\
\hline \multirow[t]{3}{*}{ RTFOT (163ㅇ, $85 \mathrm{~min})$} & Loss of quality/\% & $\ngtr \pm 0.8$ & 0.07 \\
\hline & Penetration ratio/\% & $\nless 54$ & 70 \\
\hline & Ductility at $10^{\circ} \mathrm{C} / \mathrm{cm}$ & $\nless 6$ & 9.0 \\
\hline
\end{tabular}

(5) The segregation tube is cut into upper, middle, and lower sections, in preparation for the test.

The segregation test sample can be seen in Figure 1, and asphalts modified by various rubber powder content $(20 \%, 25 \%$, $30 \%, 40 \%, 50 \%, 60 \%$, and $70 \%$ ) were used.

\section{Dynamic Mechanical Method}

The dynamic shear rheological (DSR) test is widely accepted in studying the rheological properties of asphalts and has the advantages of easy operation and high testing precision. The evaluation indexes of DSR are complex shear modulus $G^{*}$ and phase angle $\delta$. Recently, the DSR method was introduced to analyze the storage ability of rubber powder-modified asphalt by comparing the rheological property differences of modified asphalts between upper and lower sections of the segregation tube (Bahia et al., 1998). In this study, the rheological property differences in the upper and lower sections of various asphalts were assessed utilizing the DSR method, and the storage abilities of tested asphalts were analyzed.

\section{Laboratory Asphalt Stability Test (LAST)}

The LAST is a part of the National Highway Cooperation Research Program (NCHRP) 9-10 project, and it was developed to study the storage stability of asphalt binders modified by elastomers, plastomers, and solid additives (Polacco et al., 2015). This method involves the use of a small storage cabinet composed of an internal heater, an external heater, an agitator, and a temperature control device, which was designed to simulate the actual storage conditions.

\section{Fluorescence Imaging Method}

A fluorescence microscope (FM) was used to scan the target with $420 \mathrm{~nm}$ wavelength monochromatic light or $365 \mathrm{~nm}$ wavelength ultraviolet and observe distribution properties of targets according to the fluorescence shape.

According to the mechanism of modified asphalt, the rubber powders distribute in the asphalt matrix after shear mixing. The fluorescence reaction occurs on the rubber powder when lighted by various lasers. Asphalts are black and opaque and have no fluorescence reaction. The fluorescence reaction difference between rubber powder and asphalt can be used to present the distribution properties. Figure 2 shows the FM device and the distribution of rubber powders in modified asphalt.

\section{Evaluation Index}

\section{Softening-Point Difference}

The softening-point difference between the upper and lower section separation pipe is the most commonly used method in assessing the storage stability of polymer-modified asphalt. Generally, it is considered that the storage stability of modified asphalt can meet the requirement of application if the softeningpoint difference is less than $2.5^{\circ} \mathrm{C}$.

The softening-point measurement was conducted based on the T0606-2011 in "Standard Test Methods of Bitumen and Bituminous Mixtures for Highway Engineering" (JTG E202011, 2011), and the softening-point difference can be calculated according to the following equation:

$$
S_{\text {diff }}=\left|S_{t}-S_{b}\right|
$$

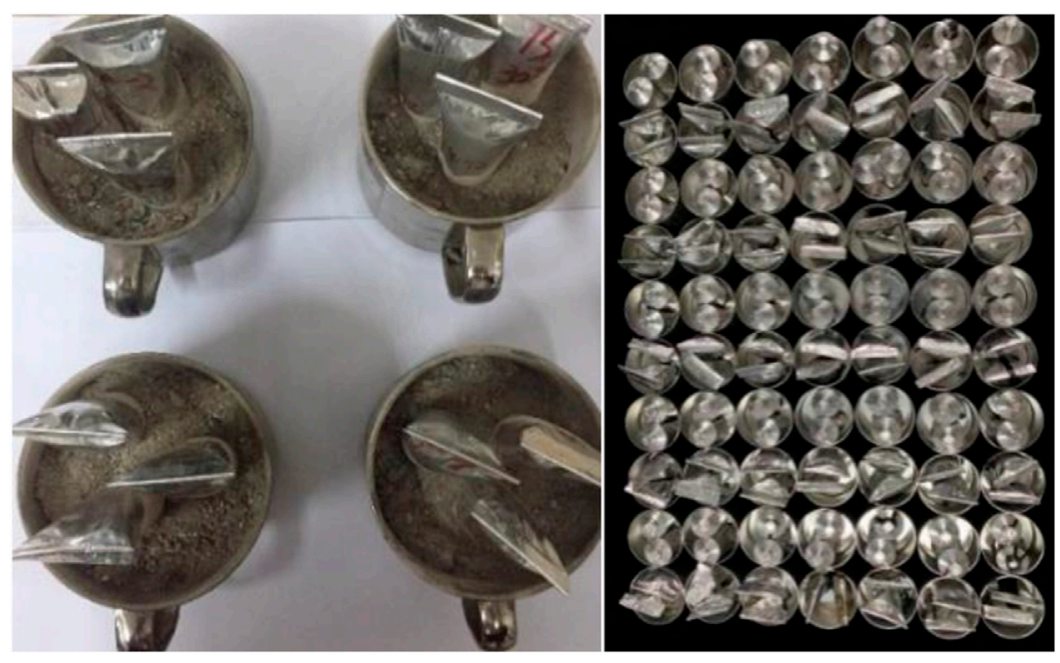

FIGURE 1 | Segregation test 


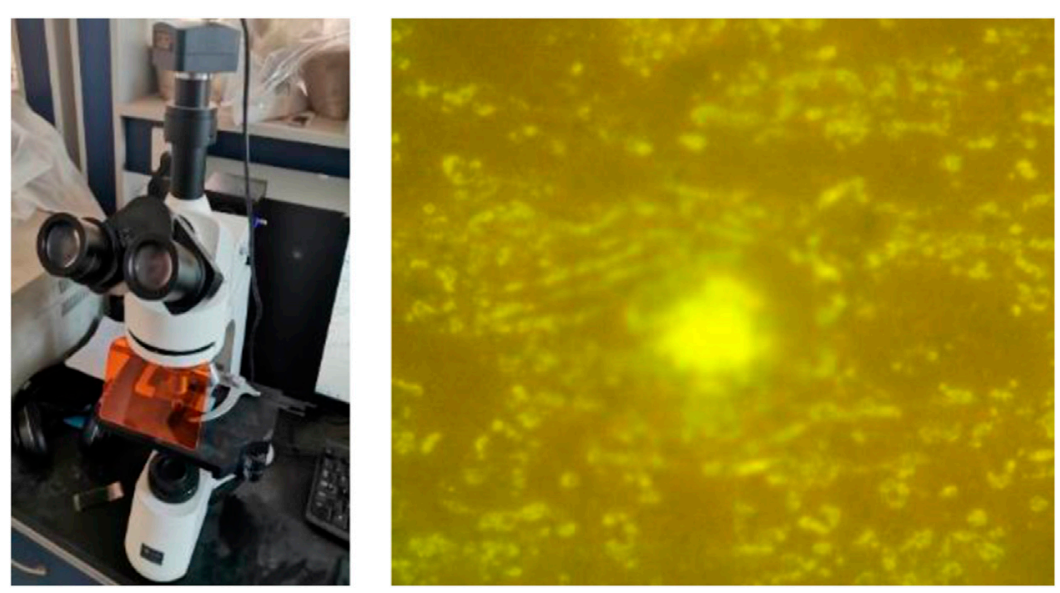

FIGURE 2 | Fluorescence microscope and fluorescence micro-images.

where $S_{t^{-}}$is the softening-point value of the upper part of the separation tube, ${ }^{\circ} \mathrm{C}$, and $\mathrm{S}_{b^{-}}$is the softening-point value of the lower part of the separation tube, ${ }^{\circ} \mathrm{C}$.

\section{Segregation Percentage}

The segregation percentage is used to evaluate the storage stability of polymer-modified asphalt. This is a recently developed evaluation method based on the ASTM D 5892-00 (Bahia and Zhai, 2000; ASTM D5892-00, 2001). The complex shear modulus $G^{*}$ of the upper and lower sections of the segregation test is measured at high temperature utilizing the DSR method ( $25 \mathrm{~mm}$ parallel plate, $54^{\circ} \mathrm{C}$ testing temperature, and $10 \mathrm{rad} / \mathrm{s}$ frequency), and then the segregation percentage can be calculated according to Eq. 2. A smaller segregation percentage means better storage stability.

$$
S_{p}=\frac{\max \left(G_{t}^{*}, G_{b}^{*}\right)-G_{\text {avg }}^{*}}{G_{\text {avg }}^{*}} \times 100,
$$

where $G_{t}{ }^{*}$ and $G_{b}{ }^{*}$ are the complex shear modulus values of the upper and lower sections of the separation tube and $G^{*}$ avg is the average value of the complex shear modulus of the upper and lower sections.

\section{Segregation Index}

The segregation index $\left(I_{s e}\right)$ is calculated based on the logarithm of complex shear modulus ratio, which can be used to evaluate the storage stability of polymer-modified asphalt (Li et al., 2019), and $I_{s e}$ can be calculated according to Eq. 3. It can be concluded that a smaller absolute value of $I_{s e}$ means better storage stability of modified asphalt. The complex shear modulus in this research was acquired with the testing parameters of an $8 \mathrm{~mm}$ parallel plate, $25^{\circ} \mathrm{C}$ testing temperature, and $10 \mathrm{rad} / \mathrm{s}$ frequency.

$$
I_{s e}=\log \left(\frac{G_{b}^{*}}{G_{t}^{*}}\right) \text {. }
$$

\section{Segregation Rate and Degradation Rate}

The complex shear modulus $G^{*}$ and phase angle $\delta$ can be used to calculate the segregation rate and degradation rate at high and medium temperatures. The storage stability of the asphalt binder was evaluated using Eqs. 4 and $\mathbf{5}$ to calculate the segregation rates of samples at different times over $48 \mathrm{~h}$, and the same data were also used to calculate degradation ratios according to Eqs. 6 and 7. It is considered that segregation or degradation occurs if the index value is out of the range of $0.8-1.2$.

$$
\begin{gathered}
R_{s H T}=\frac{\left(G^{*} / \sin \delta\right)_{t}}{\left(G^{*} / \sin \delta\right)_{b}}, \\
R_{s I T}=\frac{\left(G^{*} / \sin \delta\right)_{t}}{\left(G^{*} / \sin \delta\right)_{b}}, \\
R_{d H T}=\frac{\left(G^{*} \cdot \sin \delta\right)_{t}+\left(G^{*} \cdot \sin \delta\right)_{b}}{2\left(G^{*} \cdot \sin \delta\right)_{i n}}, \\
R_{d I T}=\frac{\left(G^{*} / \sin \delta\right)_{t}+\left(G^{*} / \sin \delta\right)_{b}}{2\left(G^{*} / \sin \delta\right)_{i n}},
\end{gathered}
$$

where $G^{*} / \sin \delta$ is the rutting factor and $G^{*} \cdot \sin \delta$ is the fatigue factor. $H T$ and IT represent the high and medium temperature, respectively; $t$ and $b$ represent the upper and lower sections of the separation tube, respectively; and in represents the initial value.

\section{Stability Index}

The fluorescence area of rubber powders in the asphalt matrix was analyzed using Image Pro Plus software. The stability index $\left(I_{s t}\right)$ can be calculated according to Eq. 8. $I_{s t}$ can evaluate the storage ability of modified asphalts based on the morphology method.

$$
I_{s t}=\frac{S_{t}}{S_{b}},
$$

where $S_{t}$ is the fluorescence area of rubber powder in upper section and $S_{b}$ is the fluorescence area of rubber powder in lower section. 


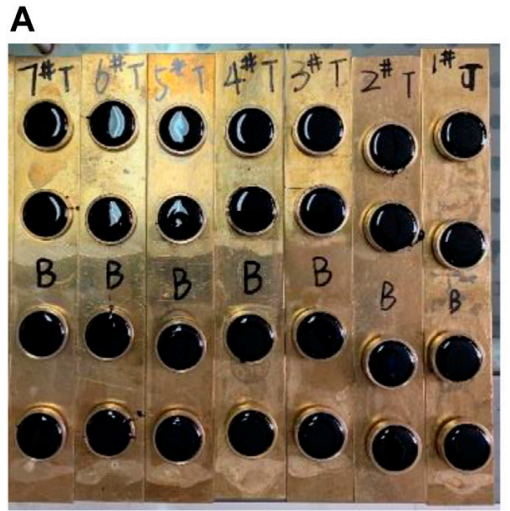

Specimen

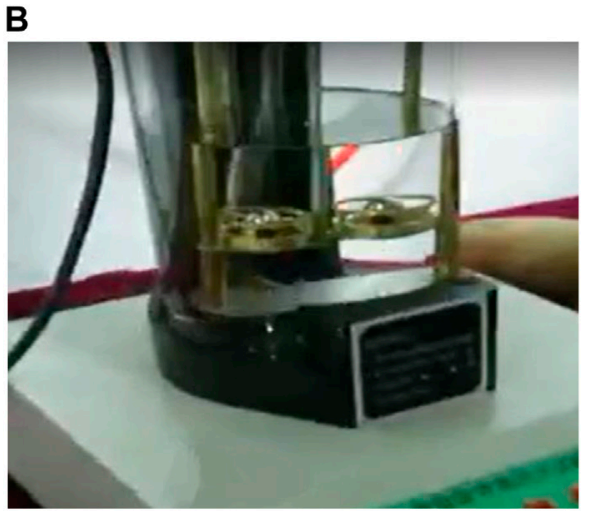

Testing device

FIGURE 3 | Softening-point test. (A) Specimen. (B) Testing device.

\section{RESULTS AND ANALYSIS}

\section{Softening-Point Difference}

To study the softening-point difference between the upper and lower sections of the segregation tube, asphalts with various activated rubber powder contents of $20 \%, 25 \%$, $30 \%, 40 \%, 50 \%, 60 \%$, and $70 \%$ were employed. To further understand the effect of storage time, the softening-point difference asphalts $(50 \%, 60 \%$, and $70 \%)$ from the upper and lower sections of the segregation pipe were measured after pouring for $1,2,3,4$, and $5 \mathrm{~h}$, and four replicates were tested for each parameter.

Figure 3 shows the poured softening-point specimen and the softening-point measurement, using a softening-point meter, and Figure 4 presents the developing rules of softening-point difference with various rubber powder contents.

As can be seen in Figure 4, with the increase in the activated rubber powder content, the softening-point difference gradually decreases. The softening-point difference reaches its maximum at $20 \%$ and its minimum at $70 \%$. It can be concluded that the storage

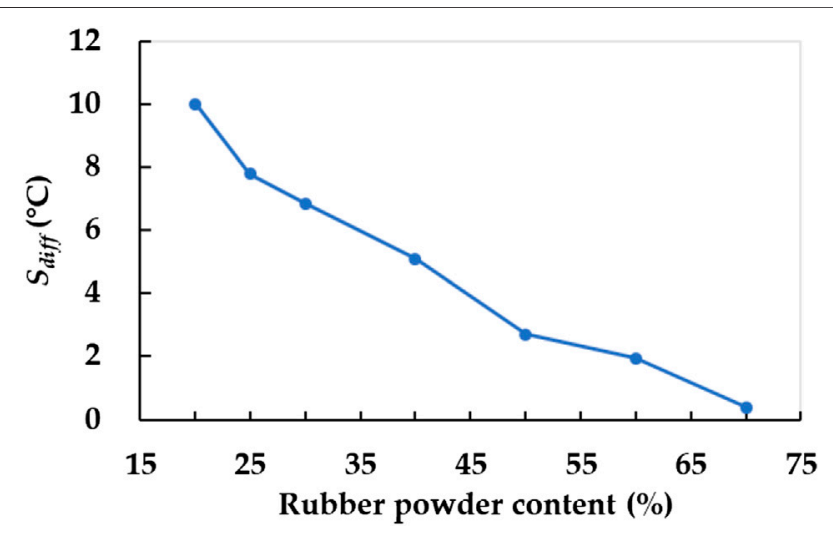

FIGURE 4 | Variation of softening-point difference with content. stability of $20 \%$ rubber powder-modified asphalt is the worst, and that of $70 \%$ is the best.

To research the softening-point difference between the upper and lower sections of the separation pipe, the 50\%, 60\%, and $70 \%$ activated rubber powder content modified asphalts were tested at various shear times $(1,2,3,4$, and $5 \mathrm{~h})$, and the test results can be seen in Figure 5.

In Figure 5, the softening-point difference of asphalts modified with different rubber powder contents decreases as shear time increases. The overall softening-point difference curves of $50 \%$ rubber powder content asphalt are higher than those of $60 \%$, but they present a similar trend. For the asphalt of $70 \%$ rubber powder content, the curve drops rapidly in the first $2 \mathrm{~h}$ under the effect of high-speed shear loading and then stays stable in the last $3 \mathrm{~h}$.

\section{Segregation Percentage}

The developing rules of segregation percentage value of modified asphalt at the rubber powder content range from $20 \%$ to $70 \%$, as shown in Figure 6.

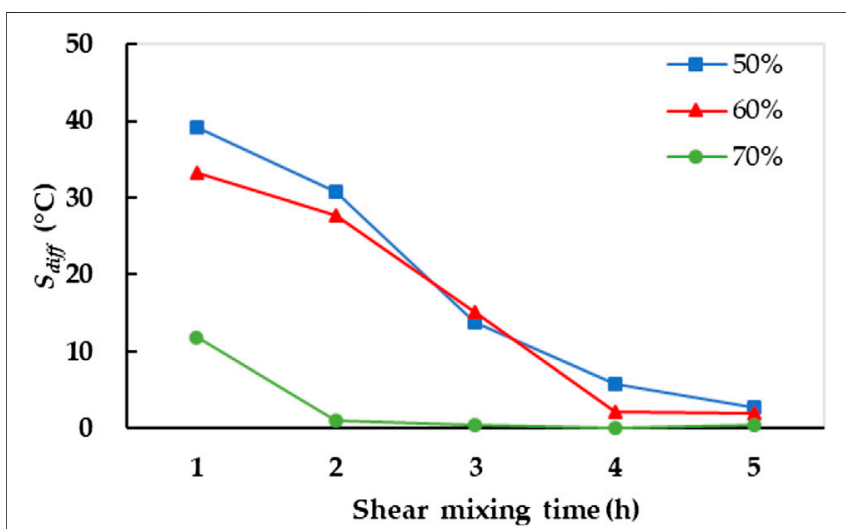

FIGURE 5 | Variation of softening-point difference of asphalt modified by high-content activated rubber powder with shear time. 


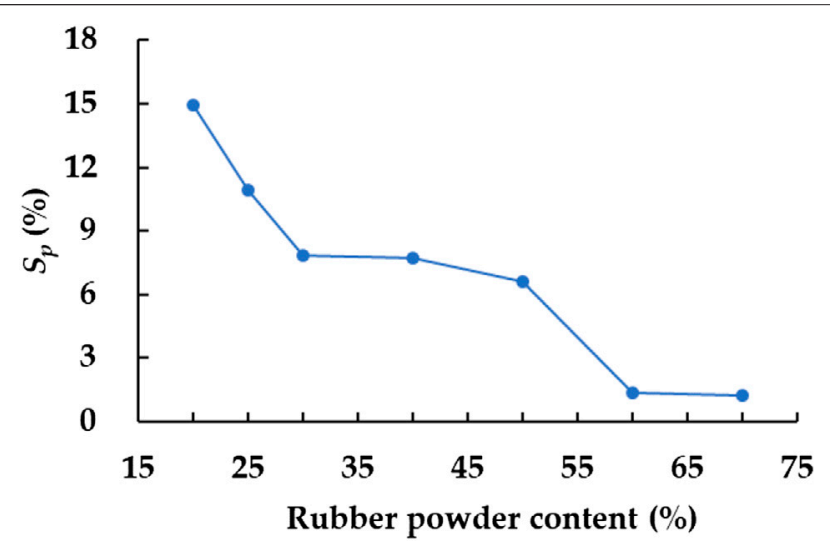

FIGURE 6 | The change of segregation percentage with dosage.

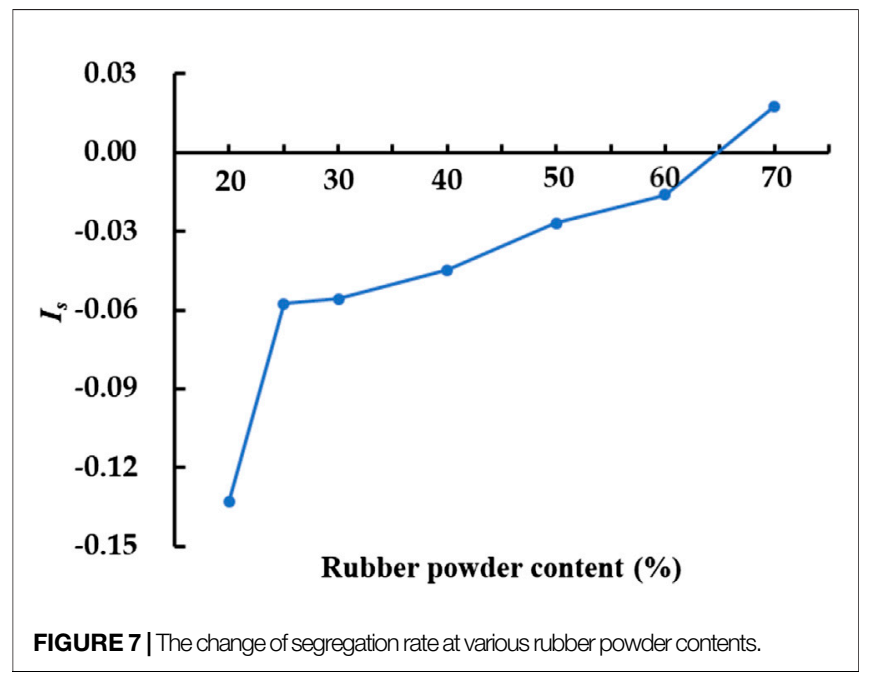

It can be seen in Figure 6 that the segregation percentage gradually decreases with the increase in rubber powder content. The segregation percentage value reached its maximum value of $14.92 \%$ at the rubber powder content of $20 \%$ and reached its minimum value of $1.24 \%$ at the rubber powder content value of $70 \%$. Two inflection points can also be seen at the rubber powder contents of $30 \%$ and $60 \%$ in the curve.

\section{Segregation Index}

The developing rules of segregation rate at various rubber powder contents can be seen in Figure 7.

It can be seen from Figure 7 that the segregation index $\left(I_{s e}\right)$ increases gradually along with the increase in activated rubber powder content. The segregation index of $20 \%$ rubber powdermodified asphalt has the biggest absolute value among all. The absolute values of $60 \%$ and $70 \%$ are the two lowest, and the zero point can be found between $60 \%$ and $70 \%$ rubber powder content.

\section{Segregation Rate and Degradation Rate}

The high-temperature and medium-temperature segregation rates of different modified asphalts with rubber powder content varying from $20 \%$ to $70 \%$ can be seen in Figures 8A and B.

Figure 8A illustrates that, with the increase in the rubber powder content, the segregation rate increases from 0.32 to 0.99 . According to the specification of the LAST, the standard segregation rate is required to vary from 0.8 to 1.2 . It can be seen that asphalts modified by $60 \%$ and $70 \%$ rubber powder contents are applicable in actual working condition.

In Figure 8B, the segregation rates at medium temperature of tested asphalts are all between 0.8 and 1.2, and it can be concluded that the segregation rate index is not sensitive to the variation of rubber powder content in modified asphalt. Combining Figures 8A and $\mathbf{8 B}$, the rubber powder content between $60 \%$ and $70 \%$ presents the best storage ability in both conditions.

The high-temperature and medium-temperature degradation rates of different modified asphalts with rubber powder content varying from 20\% to $70 \%$ can be seen in Figures 9A and 9B.

In Figure 9, the degradation rates of all the tested modified asphalts in high and medium temperatures are between 0.8 and 1.2 , and there is no clear tendency along with the increases of rubber powder content. It can be concluded that the degradation rate index cannot differentiate the difference among asphalts with various rubber powder contents significantly.

\section{Stability Index}

A fluorescence microscope (FM) was employed to research the microscopic rubber powder distribution properties of modified asphalts under high-temperature and high-speed shear mixing, and the observation results of $50 \%, 60 \%$, and $70 \%$ activated rubber powder content asphalts can be seen in Figure 10.

It can be seen in Figure $\mathbf{1 0}$ that the rubber powder distribution density of various asphalts is relatively large.
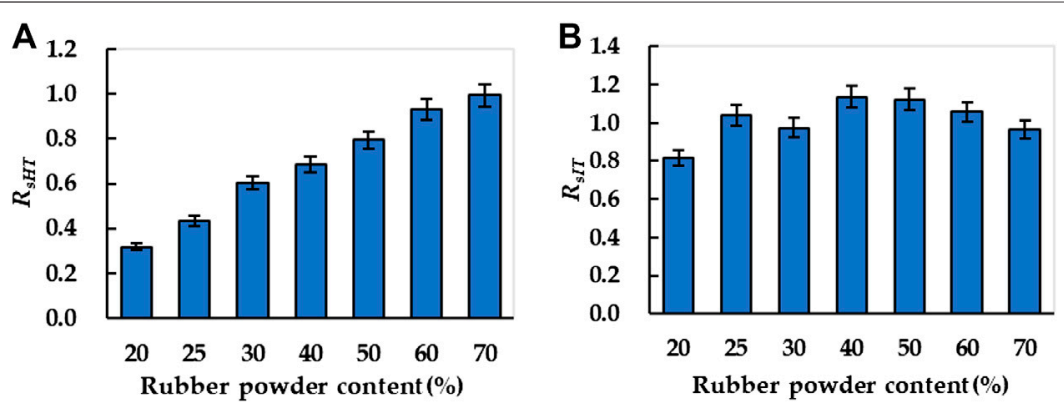

FIGURE 8 | The variation of segregation rate with the amount of the rubber powder. (A) High temperature. (B) Medium temperature. 

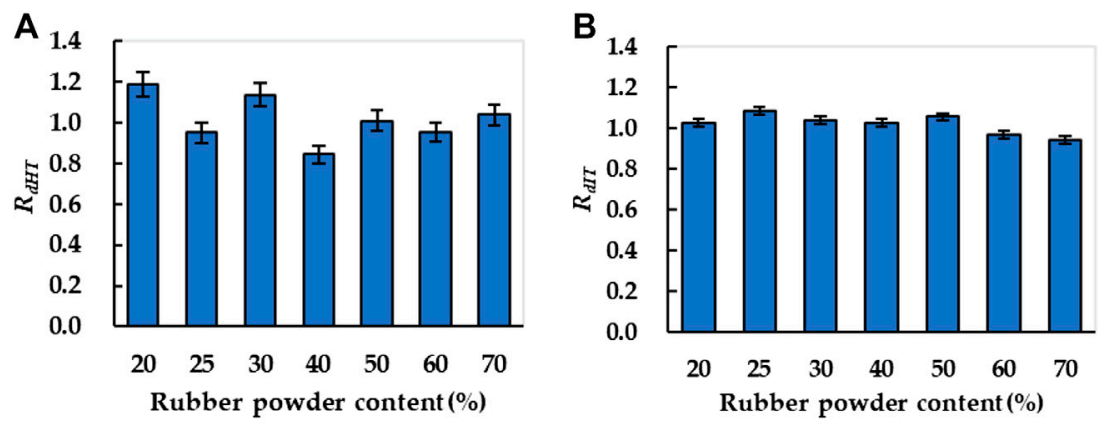

FIGURE 9 | The variation of degradation rate with the amount of rubber powder. (A) High temperature. (B) Medium temperature.

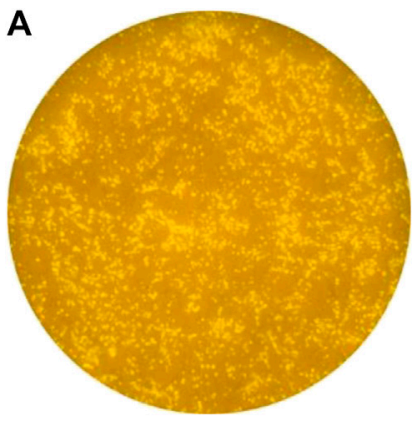

$50 \%$

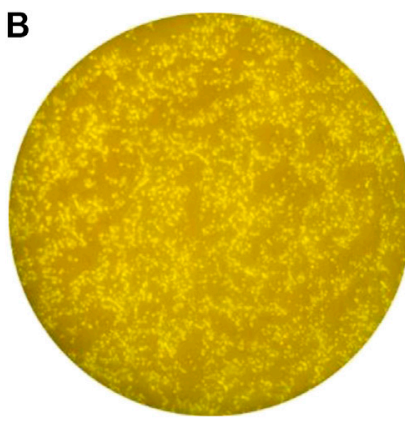

$60 \%$

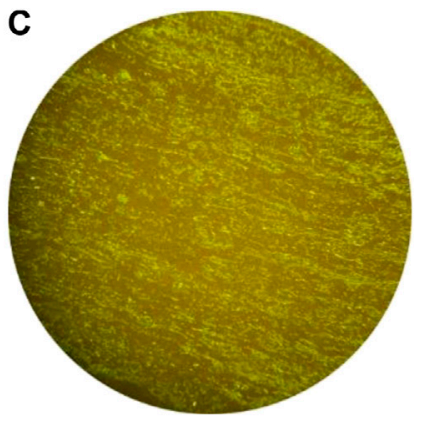

$70 \%$

FIGURE 10 | Fluorescence images of asphalt modified by high-content activated rubber powder. (A) 50\%, (B) 60\%, and (C) $70 \%$.

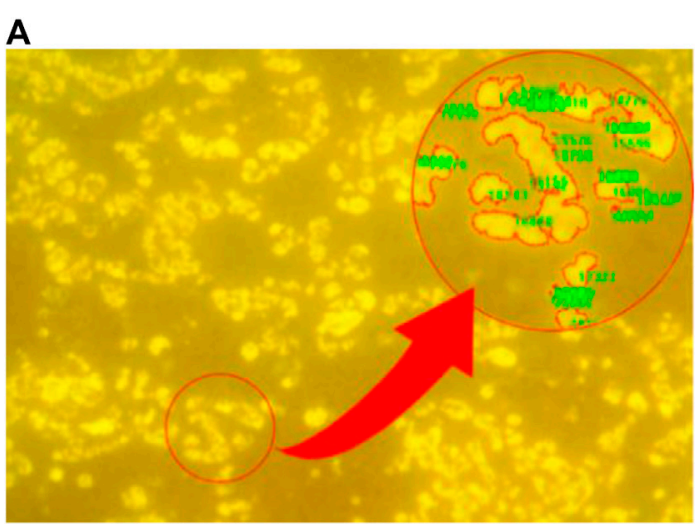

Analysis principles
B

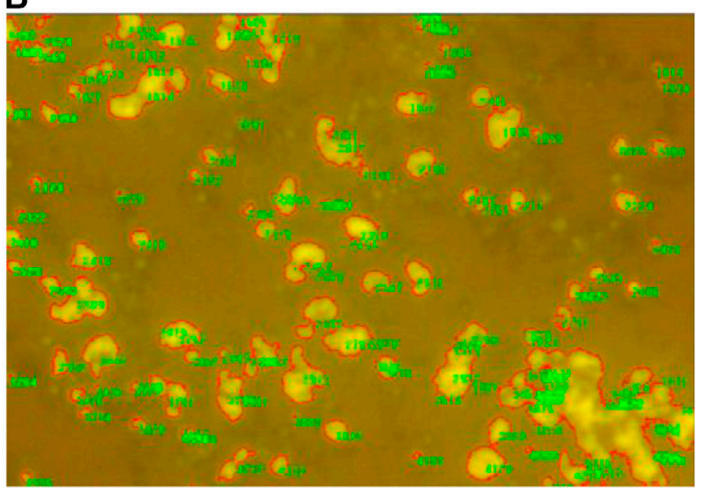

Analysis details

(B) Analysis details.

Among them, the $50 \%$ and $60 \%$ rubber powder content asphalts have similar morphology, but the rubber powder distribution density of $60 \%$ asphalt is higher than that of $50 \%$ asphalt. For $70 \%$ asphalt, the rubber powder distribution structure in the matrix changes, and small crosslinking phases can be found. It can be concluded that there is not enough light component in asphalt, and the rubber powder cannot be dispersed sufficiently.
To further understand the micro-distribution properties of modified asphalt quantitatively, Image Pro Plus software was utilized to analyze the fluorescent images, as shown in Figure 11.

Based on the fluorescence microscopy method and IPP software, the upper and lower section in the separation pipe of $50 \%, 60 \%$, and $70 \%$ rubber powder content asphalts were measured, and the stability indexes were calculated to indicate the storage abilities of 


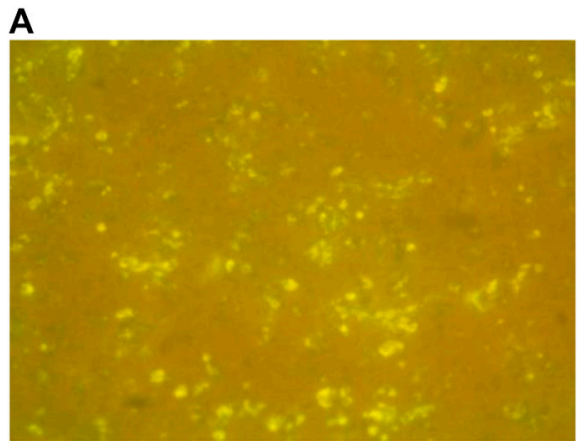

$50 \%$ upper segment

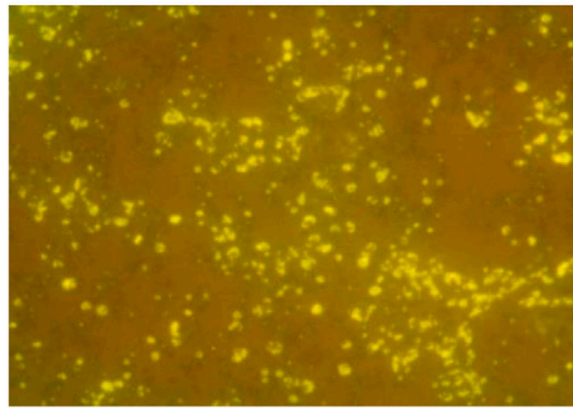

$60 \%$ upper segment

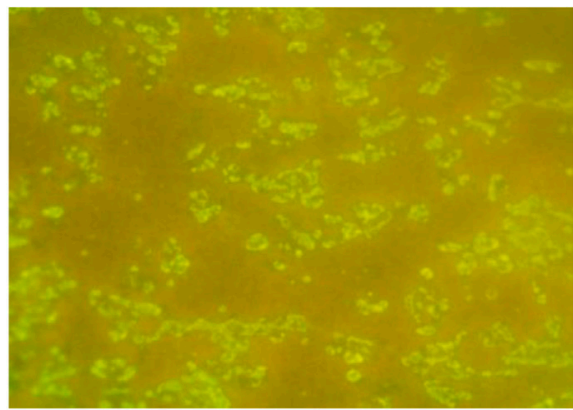

$70 \%$ upper segment

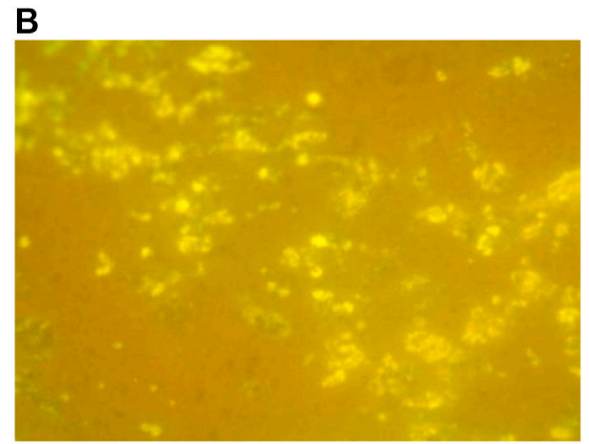

$50 \%$ lower segment

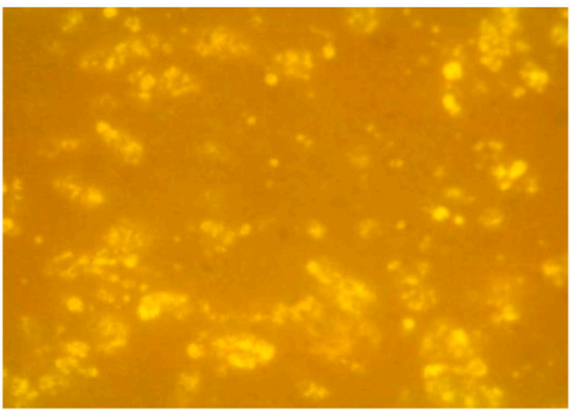

$60 \%$ lower segment

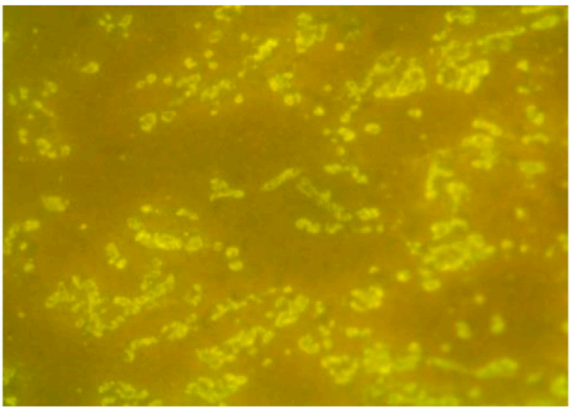

$70 \%$ lower segment

FIGURE 12 |Fluorescence images of various modified asphalts. (A) 50\% upper segment, 60\% upper segment, and $70 \%$ upper segment. (B) $50 \%$ lower segment, $60 \%$ lower segment, and $70 \%$ lower segment.

TABLE 3 | Stability index of various modified asphalts $\left(I_{s t}\right)$.

\begin{tabular}{|c|c|c|c|c|c|}
\hline \multirow{2}{*}{$\begin{array}{l}\text { Rubber } \\
\text { powder content (\%) }\end{array}$} & \multicolumn{2}{|c|}{ Total area $\mathbf{S}$} & \multicolumn{2}{|c|}{ Maximum area $M$} & \multirow[t]{2}{*}{ Stability index $I_{\text {st }}$} \\
\hline & Upper section & Lower section & Upper section & Lower section & \\
\hline 50 & 472,568 & 500,346 & 47,116 & 53,205 & 0.944 \\
\hline 60 & 533,086 & 560,972 & 50,826 & 55,486 & 0.950 \\
\hline 70 & 583,957 & 619,645 & 52,909 & 60,914 & 0.942 \\
\hline
\end{tabular}

various asphalts. The fluorescence images and stability indexes of the three tested asphalts can be seen in Figure $\mathbf{1 2}$ and Table 3.

It can be seen from Figure $\mathbf{1 2}$ that the rubber powders agglomerate when their content is as large as $70 \%$, especially in the lower segment. In Table 3, the stability index $\left(I_{s t}\right)$ of the $70 \%$ asphalt is the lowest among all, which shows that the rubber powder content of $70 \%$ exceeds the optimum proportion of modified asphalt. The rubber powder content of $60 \%$ has the highest $I_{s t}$ value, and it has the best rubber powder distribution structure. Therefore, it can be concluded that asphalt modified by the $60 \%$ rubber powder content presents the best storage ability. 


\section{DISCUSSION}

Ground tire rubber can be utilized to modify asphalts in sustainable pavement construction and provide an effective method to diminish the effects of urban solid waste. However, the storage problems of asphalts modified by ordinary rubber powder restrict its further application, and the storage ability decreases as the rubber powder content increases for the ordinary modified asphalts.

According to this research, the storage abilities of asphalts modified by activated rubber powder have a positive correlation with rubber powder content, which presents a different tendency than the asphalts modified by ordinary rubber powder. The reasons can be explained by two main factors as follows. Firstly, the low-content activated rubber powder can disperse uniformly in the asphalt matrix but settles easily at the bottom of segregation tube in heated storage conditions. This is due to the low viscosity of asphalt caused by the existence of waste oil. Secondly, the activated rubber powder is prepared based on the combination of microwave treatment and waste oil pre-swelling, which improves its compatibility and distribution properties in the asphalt matrix.

In Figure 5, the softening-point differences of 50\% and $60 \%$ rubber powder content modified asphalts decrease over shear mixing time. It can be concluded that the activated rubber powders depolymerized during shear mixing and formed a stable distribution structure. The $60 \%$ rubber powder content asphalt presents better distribution properties compared to that of $50 \%$. For the $70 \%$ rubber powder content asphalt, the curve tends to flatten after shear mixing for $2 \mathrm{~h}$, which means that the swelling process is finished in the first $2 \mathrm{~h}$, and there is not enough light component in the asphalt matrix. The rubber powder distributed in asphalt exceeds the optimum dosage.

Based on the segregation percentage test results in Figure 6, the best two rubber powder contents are $60 \%$ and $70 \%$ compared with the five other groups. There is also an inflection point at $60 \%$ content, after which the storage ability of modified asphalt has no significant improvement.

As shown in Figure 7, the segregation index was employed to indicate segregation level of modified asphalt. Based on Eq. 3, asphalt modified by $60 \%$ rubber powder content has the lowest absolute value among all, indicating that $60 \%$ rubber content has the optimal distribution structure. In addition, the absolute value of various modified asphalts presents an overall decreasing trend with the increase in rubber powder content, which agrees well with the conclusions of the softening-point difference tests.

Based on the segregation rate and degradation rate shown in Figures $\mathbf{8}$ and $\mathbf{9}$, only the segregation rate at high temperature presents a clear tendency for various rubber powder contents, and $60 \%$ and $70 \%$ rubber powder contents can meet the requirement of the LAST specification. The difference among modified asphalts cannot be differentiated significantly, and the evaluation results have no clear correlation with the other tests. It can be seen that the evaluation indexes are not applicable in this study.

According to the fluorescence microscope (FM) scanning results in Figure 10, the rubber powders in 50\% and $60 \%$ modified asphalts distribute uniformly, but for $70 \%$ modified asphalt, rubber powder agglomeration is formed, and the boundaries of rubber powder are not clear. In addition, the stability indexes $\left(I_{s t}\right)$ of various modified asphalts were calculated using Image Pro Plus software, and the $60 \%$ rubber powder content has the highest $I_{s t}$ value among all, which indicates that asphalt modified by $60 \%$ rubber powder content has the best storage ability. The FM scanning results are in close accordance with the other test conclusions.

Therefore, the activated rubber powder-modified asphalt provides an effective method to solve the waste ground tire problem, but the storage ability of modified asphalt needs to be researched. In this study, $60 \%$ activated rubber powder content is proven to have the best storage properties.

\section{CONCLUSION}

(1) The storage properties of modified asphalts have a positive correlation with activated rubber powder content. The $60 \%$ rubber powder content is proven to be the optimum mixing amount, and more rubber powder cannot improve the storage ability of asphalt significantly. The modified asphalts are in good homogenization condition after shear mixing for $5 \mathrm{~h}$.

(2) The evaluation results of softening-point difference, segregation percentage, and segregation index are all in good agreement with each other, but the segregation rate and degradation rate cannot differentiate the storage ability differences among various modified asphalts significantly.

(3) The rubber powders can distribute uniformly in the asphalt matrix when their contents are below $60 \%$. For the content of $70 \%$, rubber powder agglomeration is formed, and the boundaries of rubber powder are not clear. Based on the quantitative morphology analysis, asphalts modified by $60 \%$ rubber powder content have the largest stability index, which indicates their superior storage ability. The FM analysis results are in accordance with the conclusions of the laboratory test.

(4) The activated rubber powders have better compatibility with asphalts and present better storage properties in asphalt compared to the ordinary rubber powder asphalts. This provides a promising method to solve the waste ground tire problems.

\section{DATA AVAILABILITY STATEMENT}

The original contributions presented in the study are included in the article/Supplementary Material, further inquiries can be directed to the corresponding author.

\section{AUTHOR CONTRIBUTIONS}

The authors contributed to the present study as follows: HX and YC: conceptualization; HX and QL: methodology; YC, HZ, and MZ: validation; HX: formal analysis, writing-original draft 
preparation, and funding acquisition; YC: investigation and writing-review and editing; QL: resources, software, and visualization; $\mathrm{HZ}$ and MZ: data curation; HZ: supervision; MZ: project administration. All authors have read and agreed to the published version of the manuscript.

\section{REFERENCES}

Artamendi, I., Khalid, H. A., and Page, G. C. (2006). Diffusion kinetics of bitumen into waste tyre rubber. Am. Assoc. Phys. Teach. J. 20 (3), 22-33.

ASTM D5892-00 (2001). Standard specification for type IV polymer-modified asphalt cement for use in pavement construction. West Conshohocken, PA: ASTM International.

Bahia, H. U., and Zhai, H. (2000). Storage stability of modified binders using the newly developed LAST procedure. J. Road Mat. Pavement Des. 1 (1-2), 53-73. doi:10.1080/14680629.2000.9689884

Bahia, H. U., Zhai, H., and Rangel, A. (1998). Evaluation of stability, nature of modifier, and short-term aging of modified binders using new tests: LAST, PAT, and modified RTFO. Transport. Res. Rec. 1638, 64-71. doi:10.3141/ 1638-08

Ding, X., Chen, L., Ma, T., Ma, H., Gu, L., Chen, T., et al. (2019). Laboratory investigation of the recycled asphalt concrete with stable crumb rubber asphalt binder. Construct. Build. Mater. 203, 552-557. doi:10.1016/j.conbuildmat.2019.01.114

Guo, F., Zhang, J., Pei, J., Ma, W., Hu, Z., and Guanand, Y. (2020). Evaluation of the compatibility between rubber and asphalt based on molecular dynamics simulation. Front. Struct. Civ. Eng. 14 (2), 435-445. doi:10.1007/s11709-019-0603-x

Hallmark-Haack, B. L., Hernandez, N. B., Williams, R. C., and Cochran, E. W. (2019). Ground tire rubber modification for improved asphalt storage stability. Energy Fuels 33 (4), 2659-2664. doi:10.1021/acs.energyfuels.8b03558

JTG E20-2011. (2011). Standard test methods of bitumen and bituminous mixtures for highway engineering, Beijing, China: Ministry of Transport.

Li, H., Dong, B., Zhao, D., Guo, P., and Zhang, J. (2019). Physical, rheological and stability properties of desulfurized rubber asphalt and crumb rubber asphalt. Arabian J. Sci. Eng. 44 (5), 5043-5056. doi:10.1007/s13369-0183684-2

Li, P. (2005). Study on aging performance and storage stability of SBS modified asphalt. Master's thesis, China: Chang'an University.

Liao, M., and Li, X. (2004). Stability and influencing factors of rubber powder modified asphalt. J. Petroleum Chem. Eng. Univ. 17 (4), 38-41.

Lu, Q., Huang, W., and Cai, C. (2014). Properties and application prospect of terminal blending rubber asphalt. J. Chongqing Jiaotong Univ. 33 (04), 51-55. doi:10.3969/j.issn.1674-0696.2014.04.11

Luo, P., Sun, Y., Wang, S., Wang, S., Lyu, J., Zhou, M., et al. (2020). Historical assessment and future sustainability challenges of Egyptian water resources management. J. Clean. Prod. 263, 121154. doi:10.1016/j.jclepro.2020. 121154

Ma, J., Sun, G., Sun, D., Zhang, Y., Falchetto, A. C., Lu, T., et al. (2020). Rubber asphalt modified with waste cooking oil residue: optimized preparation, rheological property, storage stability and aging characteristic. Construct. Build. Mater. 258, 120372. doi:10.1016/j.conbuildmat.2020.120372

Ma, T., Wang, H., He, L., Zhao, Y., Huang, X., Chen, J., et al. (2017). Property characterization of asphalt binders and mixtures modified by different crumb rubbers. J. Mater. Civ. Eng. 29 (7), 04017036. doi:10.1061/(ASCE)MT.19435533.0001890

Maccarrone, S. (1991). Properties of polymer modified binders and relationships to mix and pavement performance. Asph. Pav. Technol. 60 (2), 2-10.

Mu, D., Luo, P., Lyu, J., Zhou, M., Huo, A., Duan, W., et al. (2020). Impact of temporal rainfall patterns on flash floods in Hue City, Vietnam. J. Flood Risk Manag. 2020, e12668. doi:10.1111/jfr3.12668

\section{FUNDING}

This research was funded by the National Natural Science Foundation of China (51908055) and Fundamental Research Funds for the Central Universities (CN) (300102219103).

Polacco, G., Filippi, S., Merusi, F., and Stastna, G. (2015). A review of the fundamentals of polymer-modified asphalts: asphalt/polymer interactions and principles of compatibility. Adv. Colloid Interface Sci. 224, 72-112. doi:10.1016/j.cis.2015.07.010

Presti, D. L. (2013). Recycled tyre rubber modified bitumens for road asphalt mixtures: a literature review. Construct. Build. Mater. 49 (6), 863-881. doi:10. 1016/j.conbuildmat.2013.09.007

Shi, J. (2011). Study on activating modification of ground tyre rubber powder. Qingdao, China: Qingdao University of Science and Technology.

Siddika, A., Al Mamun, M. A., Alyousef, R., Mugahed Amran, Y. H., Aslani, F., and Alabduljabbar, H. (2019). Properties and utilizations of waste tire rubber in concrete: a review. Construct. Build. Mater. 224, 711-731. doi:10.1016/j. conbuildmat.2019.07.108

Sienkiewicz, M., Borzędowska-Labuda, K., Wojtkiewicz, A., and Janik, H. (2017). Development of methods improving storage stability of bitumen modified with ground tire rubber: a review. Fuel Process. Technol. 159, 272-279. doi:10.1016/j. fuproc.2017.01.049

Tan, X., Zhang, J., Guo, D., Sun, G., Zhou, Y., and Zhang, W. (2020). Preparation and repeated repairability evaluation of sunflower oil-type microencapsulated filling materials. J. Nanosci. Nanotechnol. 20 (3), 1554-1566. doi:10.1166/jnn.2020.17338

Yin, J. (2008). Reasearch on compatibility performance and anti-aged performance of activated scrap rubber powder modified asphalt. Yangzhou, China: Yangzhou University.

Yin, L., Yang, X., Shen, A., Wu, H., Lyu, Z., and Li, B. (2021). Mechanical properties and reaction mechanism of microwave-activated crumb rubber-modified asphalt before and after thermal aging. Construct. Build. Mater. 267, 120773. doi:10.1016/j.conbuildmat.2020.120773

Yu, J., Ren, Z., Yu, H., Wang, D., Svetlana, S., Korolev, E., et al. (2018). Modification of asphalt rubber with nanoclay towards enhanced storage stability. Materials 11 (11), 2093. doi:10.3390/ma11112093

Zhang, C., Yang, F., Li, Q., and Yu, Y. (2017). Research progress in storage stability of rubber powder modified asphalt. Synthetic Rubber Industry 40 (05), 409-412

Zhang, Y., Luo, P., Zhao, S., Kang, S., Wang, P., Zhou, M., et al. (2020). Control and remediation methods for eutrophic lakes in the past 30 years. Water Sci. Technol. 81 (6), 1099-1113. doi:10.2166/wst.2020.218

Zhong-Yang, G., Zhi-Yang, X. U., and Ju-Nan, S. (2018). Influence of different development time on rheological property of rubber powder modified asphalt. Northern Commun. 12, 51-54.

Conflict of Interest: The authors declare that the research was conducted in the absence of any commercial or financial relationships that could be construed as a potential conflict of interest.

The reviewer XH declared a shared affiliation with one of the authors, QL, to the handling editor at time of review.

Copyright (C) 2021 Xue, Cao, Liu, Zhang and Zhang. This is an open-access article distributed under the terms of the Creative Commons Attribution License (CC BY). The use, distribution or reproduction in other forums is permitted, provided the original author(s) and the copyright owner(s) are credited and that the original publication in this journal is cited, in accordance with accepted academic practice. No use, distribution or reproduction is permitted which does not comply with these terms. 